\title{
Effect of Metal Substrate on Electrocatalytic Property of Palladium Nanowire Array for High Performance Ethanol Electro-Oxidation
}

Chuqing Liu, Ethan Adams, Zhiyang Li, Peng Yu, Hsi-Wu Wong and Zhiyong Gu*

Department of Chemical Engineering, University of Massachusetts Lowell, Lowell, MA

01854, USA

* Corresponding author: P: (978) 934-3540; F: (978) 934-3047;

E-mail: Zhiyong_Gu@uml.edu. 
Table S1: Photon Energies of Principal K-, L-shell Emission Lines. ${ }^{1}$

\begin{tabular}{|c|c|c|c|c|c|}
\hline Element & Ka1 (keV) & La1 (keV) & Lß1(keV) & Lß2 (keV) & Ly1 (keV) \\
\hline Pd & - & 2.8386 & 2.9902 & 3.1718 & 3.3287 \\
\hline Ag & - & 2.9843 & 3.1509 & 3.3478 & 3.5196 \\
\hline C & 0.2770 & - & - & - & - \\
\hline
\end{tabular}

Table S2: Electrochemical Surface Area (ECSA) of 1.5, 3.7 and $4.5 \mu \mathrm{m}$ Pd NWAs in $0.5 \mathrm{M}$ $\mathrm{H}_{2} \mathrm{SO}_{4}$ Solution.

\begin{tabular}{|c|c|}
\hline Length of the Pd NWA $(\boldsymbol{\mu m})$ & ECSA $\left(\mathbf{m}^{2} / \mathbf{g}_{\mathbf{P d}}\right)$ \\
\hline 1.5 & 16.7 \\
\hline 3.7 & 266.7 \\
\hline 4.5 & 142.9 \\
\hline
\end{tabular}

Table S3: Cyclic Voltammetry Results of Ethanol Oxidation Activity of Pd NWA on Different Metal Substrates and the Electrical Conductivity of Pure Metals. ${ }^{2}$

\begin{tabular}{|c|c|c|}
\hline Metal substrate & $\begin{array}{c}\text { Electrical conductivity } \\
\boldsymbol{\sigma}(\mathbf{S} / \mathbf{m}) \text { at } \mathbf{2 0}{ }^{\circ} \mathbf{C}\end{array}$ & $\begin{array}{c}\text { Mass activities of Pd NWA on } \\
\text { the metal substrate } \\
\left.\mathbf{A ~ m g}^{-\mathbf{1}} \mathbf{\text { Pd}}\right)\end{array}$ \\
\hline Silver $(\mathrm{Ag})$ & $6.30 \times 10^{7}$ & 9.9 \\
\hline Copper $(\mathrm{Cu})$ & $5.56 \times 10^{7}$ & 6.0 \\
\hline Nickel $(\mathrm{Ni})$ & $1.43 \times 10^{7}$ & 3.3 \\
\hline Titanium $(\mathrm{Ti})$ & $2.38 \times 10^{6}$ & 0.8 \\
\hline
\end{tabular}


Table S4: Mass Activity ( $\mathrm{A} \mathrm{mg}^{-1}{ }_{\mathrm{Pd}}$ ) of the PdNWA from this Work vs. Pd-Based Electrocatalysts for Ethanol Electro-oxidation from References ${ }^{3-8}$.

\begin{tabular}{|c|c|c|}
\hline Electrocatalysts & Mass Activity (A mg ${ }^{-1}{ }$ ) & Reference \\
\hline Pd NWA on Ag substrate & 11.00 & This Work \\
\hline PdCuP NWs & 6.67 & $\begin{array}{l}\text { Appl. Catal. B: Environ. 2019, } \\
\text { 253, 271-277 (Lv et al.) }{ }^{3}\end{array}$ \\
\hline $\begin{array}{l}\text { PdAgCu HMSs } \\
\text { (Hollow Mesoporous } \\
\text { Nanospheres) }\end{array}$ & 5.13 & $\begin{array}{l}\text { ACS Cent. Sci. 2018, } 4, \\
1412-1419 \text { (Lv et al.) }\end{array}$ \\
\hline $\mathrm{Pd}_{40} \mathrm{Ni}_{43} \mathrm{P}_{17} \mathrm{NPs}$ & 4.95 & $\begin{array}{l}\text { Nature Comm. 2017, 8, } 14136 \\
\text { (Chen et al.) }\end{array}$ \\
\hline $\mathrm{Pd}_{73} \mathrm{Cu}_{27}$ Networks & 3.00 & $\begin{array}{l}\text { J. Mater. Chem. A 2014, 2, } \\
20933 \text { (Zhao et al.) }\end{array}$ \\
\hline $\mathrm{Pd}_{2} \mathrm{Ag}_{1}$ ultrathin sNWs & 2.84 & $\begin{array}{l}\text { Appl. Catal. B: Environ. 2019, } \\
\text { 249, 116-125 (Lv et al.) }\end{array}$ \\
\hline $\begin{array}{c}\mathrm{Pd}_{5} \mathrm{Au}_{1} \text { Nanocubes } \\
\text { (Au NPs decorated Pd } \\
\text { Nanocubes) }\end{array}$ & 1.74 & $\begin{array}{l}\text { Catal. Sci. Technol. 2016, 6, } \\
\quad 5397 \text { (Zhong et al.) }\end{array}$ \\
\hline
\end{tabular}



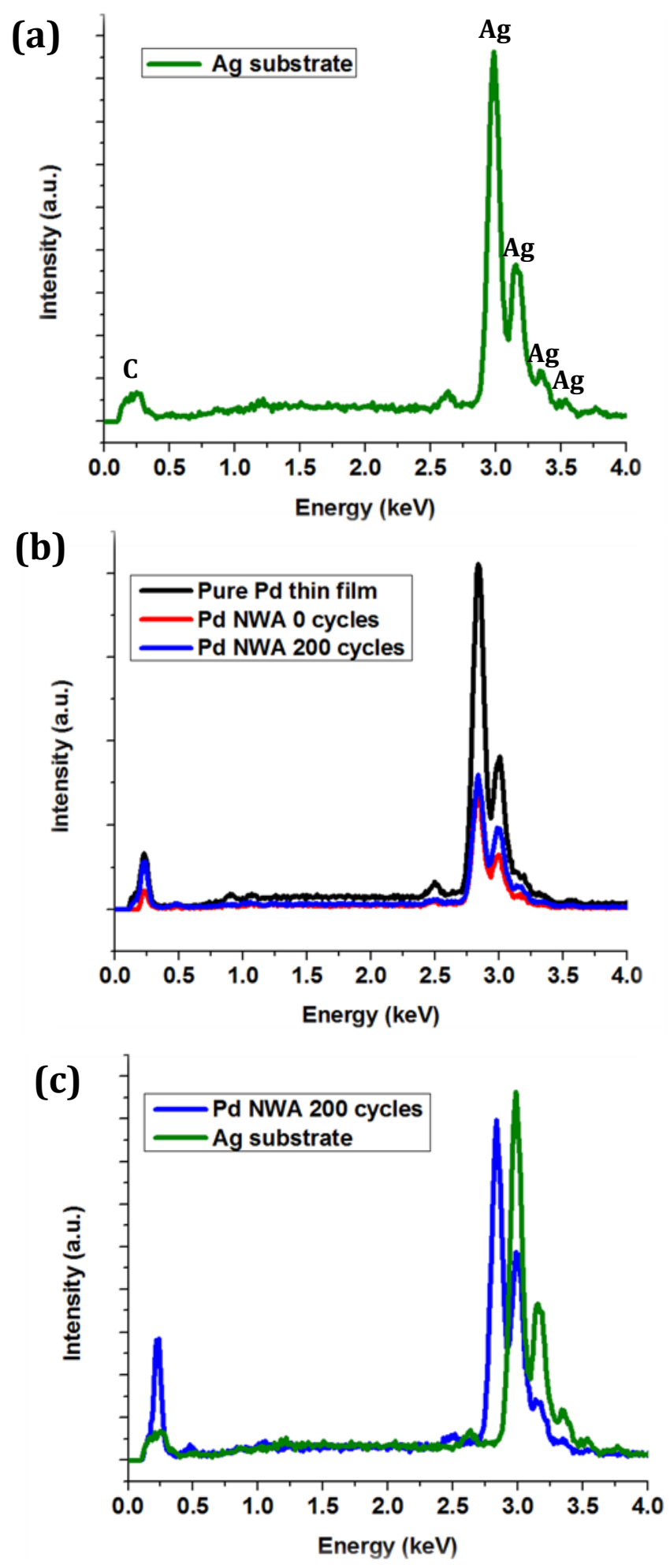

Figure S1: EDS of (a) pure Ag substrate (thin film); (b) the Pd NWA at 0 \& 200 cycles of CV scans vs. pure Pd thin film; (c) the Pd NWA after 200 cycles of CV scans vs. pure Ag substrate (thin film). 


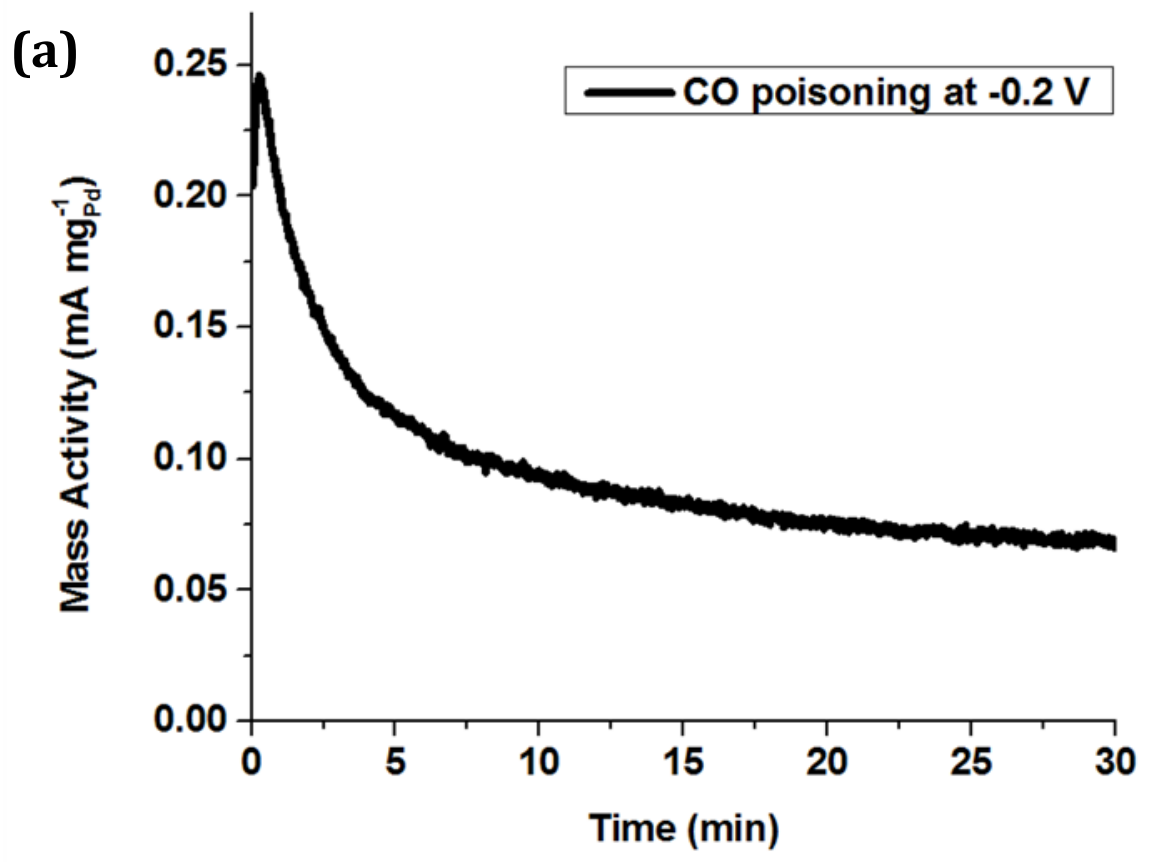

(b)

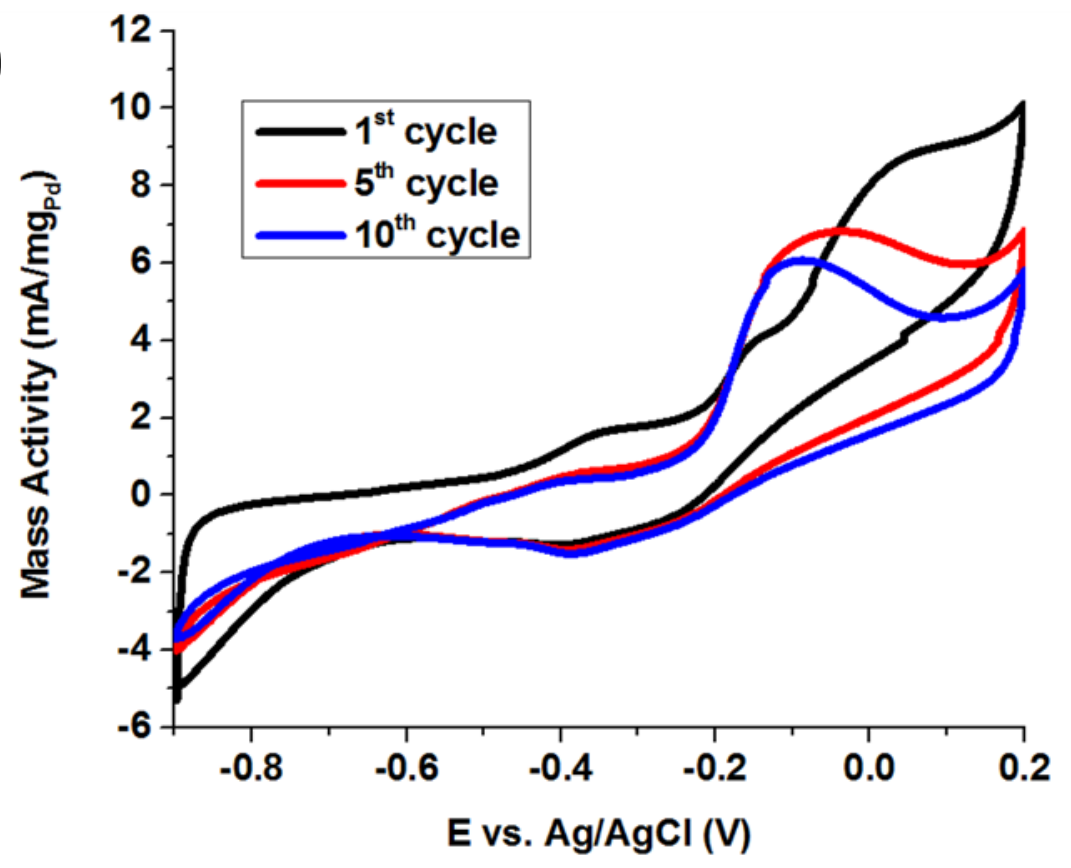

Figure S2: (a) $\mathrm{CO}$ poisoning experiment at $-0.2 \mathrm{~V}$ in $1 \mathrm{M} \mathrm{CH} \mathrm{CH}_{2} \mathrm{OH}+1 \mathrm{M} \mathrm{NaOH}$ solution for 30 minutes; (b) $\mathrm{CO}$ anti-poisoning tests between $(-0.9,0.2 \mathrm{~V})$ at a scan rate of $0.05 \mathrm{~V} / \mathrm{s}$ in $1 \mathrm{M}$ $\mathrm{NaOH}$ solution. 


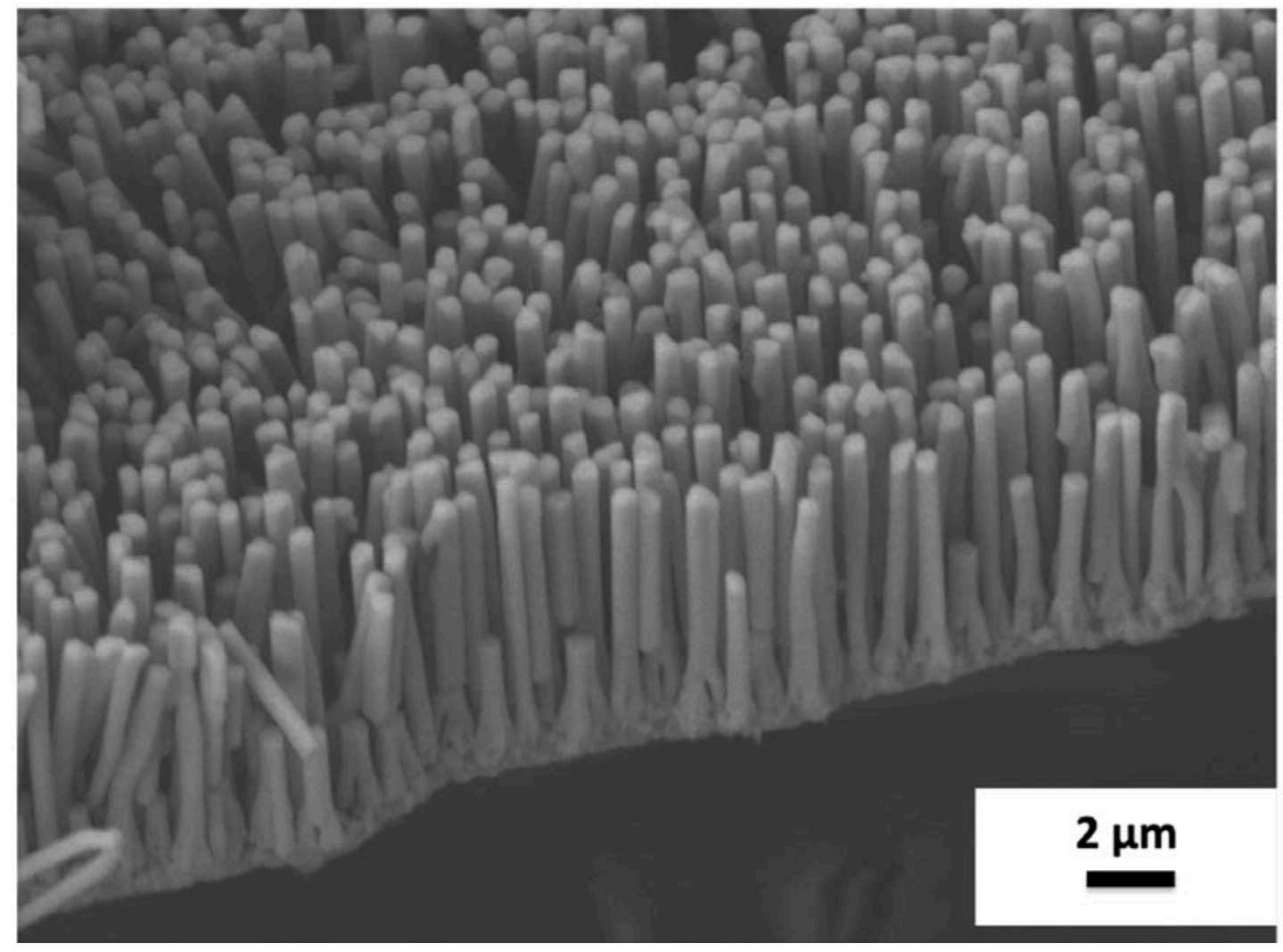

Figure S3: SEM image of Pd NWA electrode after 200 cycles of CV scans between $(-0.8,1.0 \mathrm{~V})$ at a scan rate of $0.05 \mathrm{~V} / \mathrm{s}$ in $1 \mathrm{M} \mathrm{CH}_{3} \mathrm{CH}_{2} \mathrm{OH}+1 \mathrm{M} \mathrm{NaOH}$ solution. 

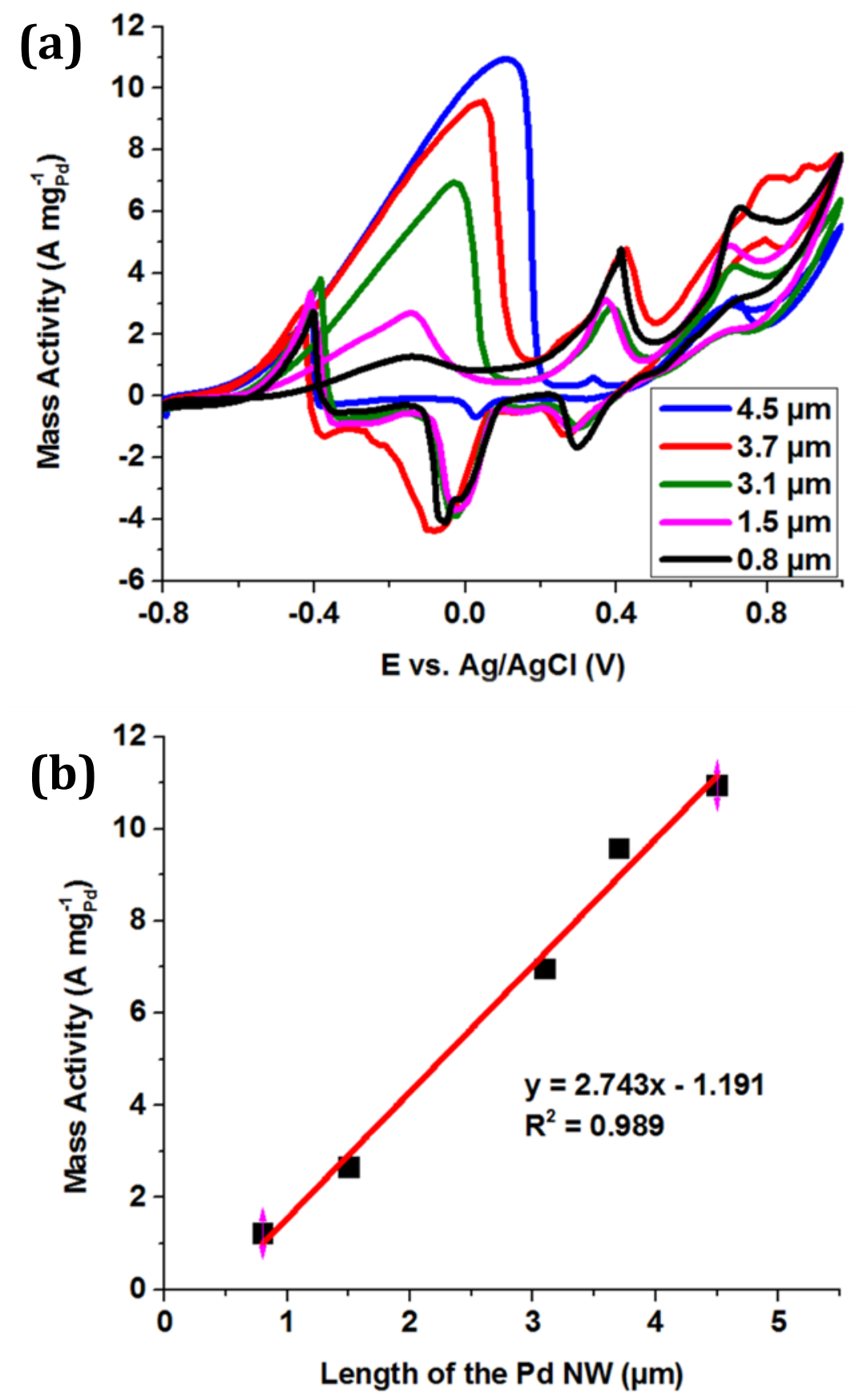

Figure S4: (a) CV plot of Pd NWAs with various lengths between $(-0.9,1.0 \mathrm{~V})$ at $0.05 \mathrm{~V} / \mathrm{s}$ in $1 \mathrm{M} \mathrm{CH}_{3} \mathrm{CH}_{2} \mathrm{OH}+1 \mathrm{M} \mathrm{NaOH}$ solution; (b) linear fit of the forward oxidation peak $\left(\mathrm{A} / \mathrm{mg}_{\mathrm{pd}}\right) \mathrm{vs}$. the length of the Pd nanowires. 

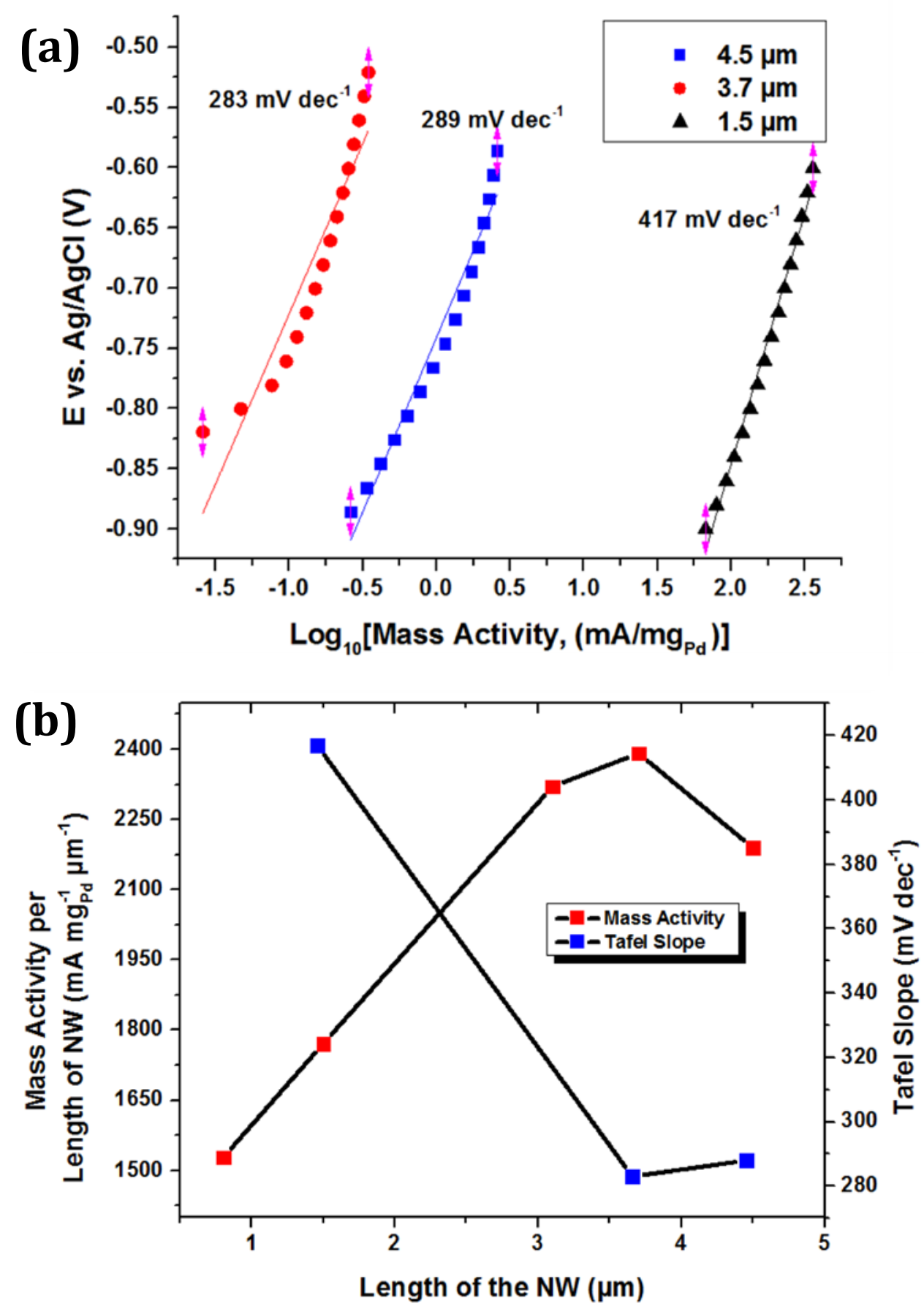

Figure S5: (a) The Tafel plot of Pd NWAs with various lengths; (b) the mass activities vs. the Tafel slopes for Pd NWs with various lengths in $1 \mathrm{M} \mathrm{CH}_{3} \mathrm{CH}_{2} \mathrm{OH}+1 \mathrm{M} \mathrm{NaOH}$ solution at a scan rate of $0.05 \mathrm{~V} / \mathrm{s}$. 


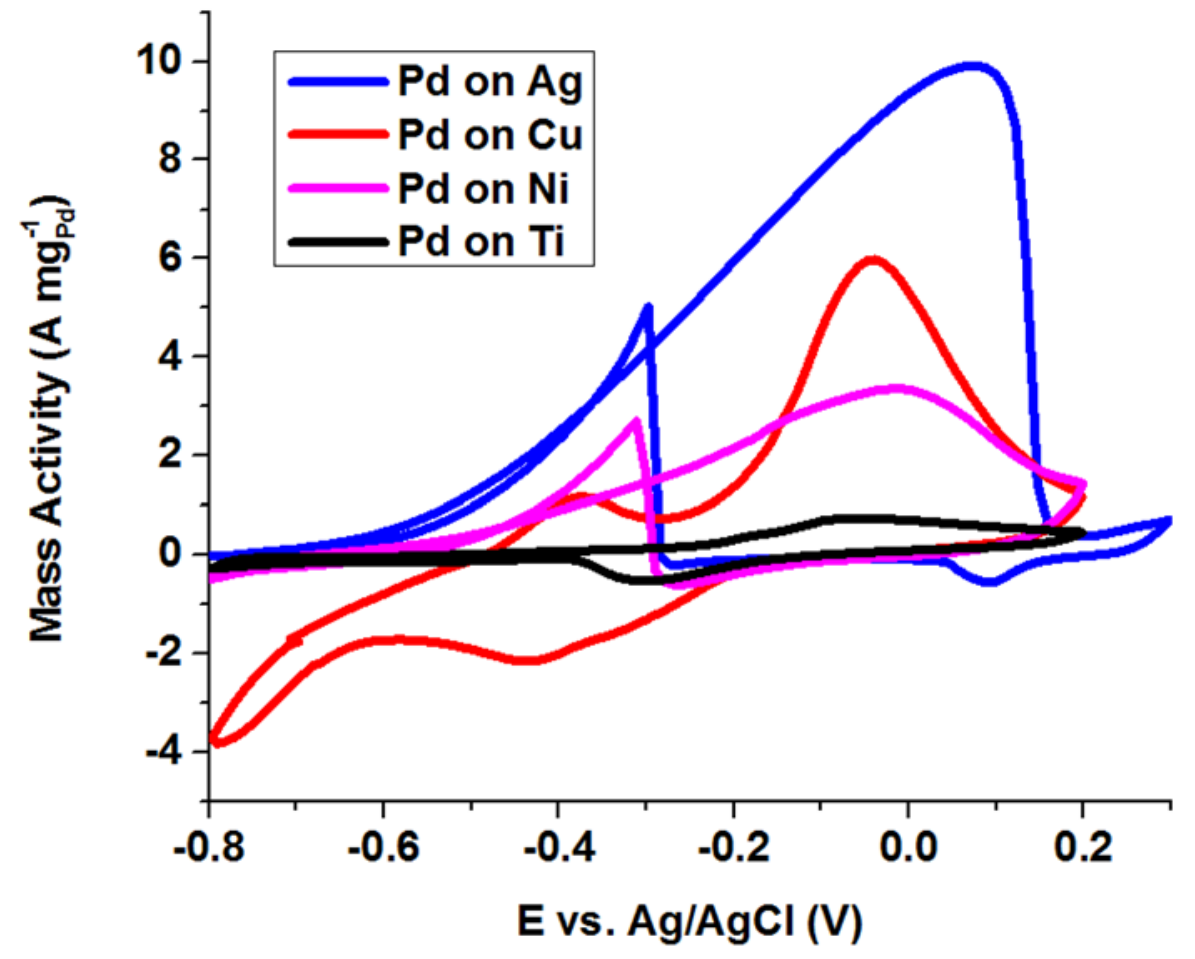

Figure S6: $\mathrm{CV}$ results for Pd NWA on $\mathrm{Ag}, \mathrm{Cu}, \mathrm{Ni}$ and Ti substrate between $(-0.8,0.3 \mathrm{~V})$ at 0.05 $\mathrm{V} / \mathrm{s}$ in $1 \mathrm{M} \mathrm{CH}_{3} \mathrm{CH}_{2} \mathrm{OH}+1 \mathrm{M} \mathrm{NaOH}$ solution during ethanol oxidation. 

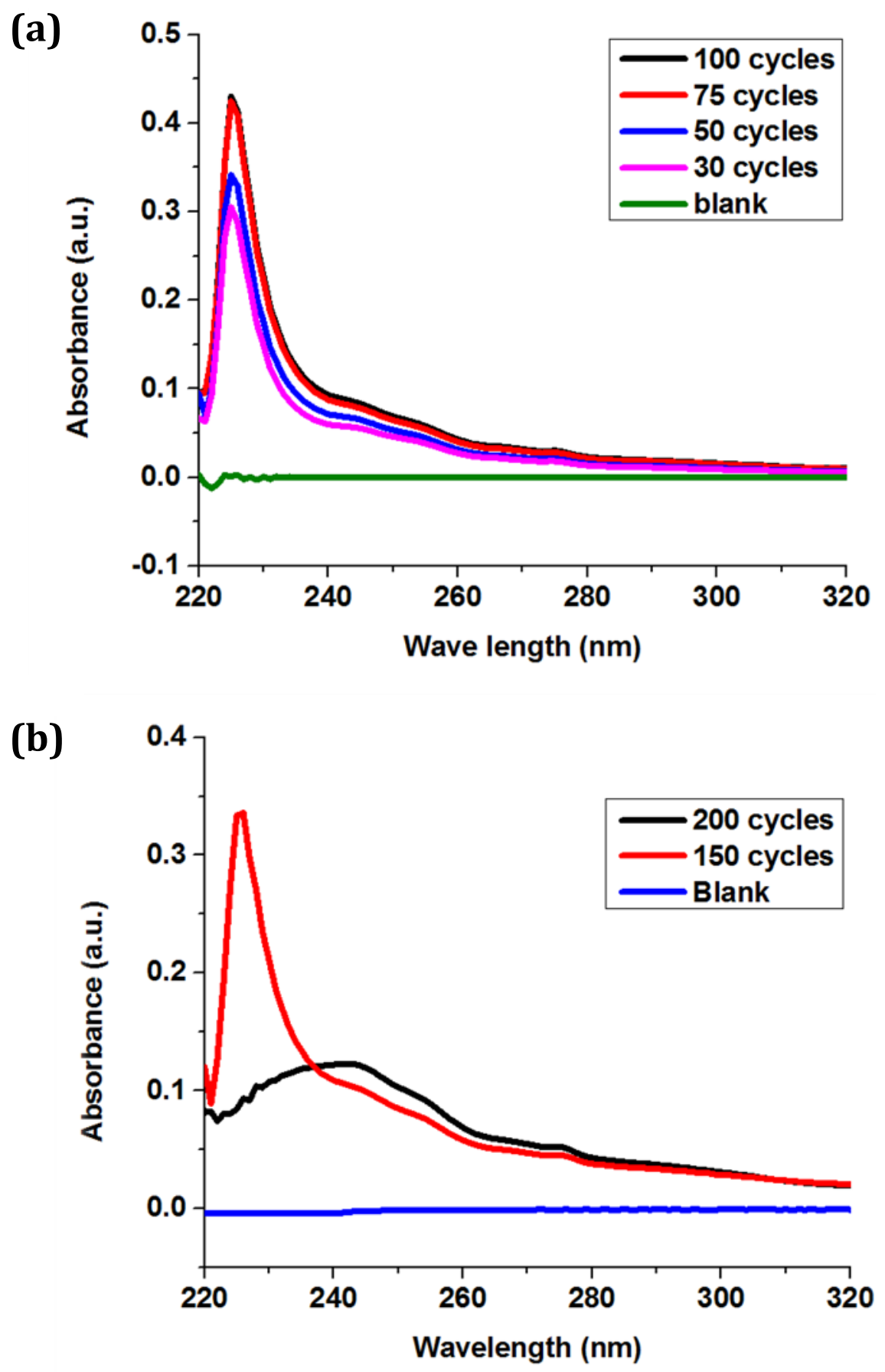

Figure S7: UV-Vis absorbance spectra for carbonate $\left[\mathrm{CO}_{2}\right.$ (aq)] (a) 30-100 cycles; (b) 150-200 cycles between $(-0.8,1.0 \mathrm{~V})$ at $0.05 \mathrm{~V} / \mathrm{s}$ in $1 \mathrm{M} \mathrm{CH}_{3} \mathrm{CH}_{2} \mathrm{OH}+1 \mathrm{M} \mathrm{NaOH}$ solution. 


\section{References}

(1) Kortright, J. B.; Thompson, A. C., X-ray data booklet. X-RAY DATA BOOKLET 2001, 35 37.

(2) Flickett, F., Electrical properties of materials and their measurement at low temperatures. U.S. Department if Commerce/National Bureau of Standards 1982, NBS Technical Note 1053, 4.

(3) Lv, H.; Sun, L.; Xu, D.; Ma, Y.; Liu, B., When ternary PdCuP alloys meet ultrathin nanowires: Synergic boosting of catalytic performance in ethanol electrooxidation. Applied Catalysis B: Environmental 2019, 253, 271-277.

(4) Lv, H.; Lopes, A.; Xu, D.; Liu, B., Multimetallic hollow mesoporous nanospheres with synergistically structural and compositional effects for highly efficient ethanol electrooxidation. ACS central science 2018, 4, 1412-1419.

(5) Chen, L.; Lu, L.; Zhu, H.; Chen, Y.; Huang, Y.; Li, Y.; Wang, L., Improved ethanol electrooxidation performance by shortening $\mathrm{Pd}-\mathrm{Ni}$ active site distance in $\mathrm{Pd}-\mathrm{Ni}-\mathrm{P}$ nanocatalysts. Nature Communications 2017, 8, 14136.

(6) Zhao, X.; Zhang, J.; Wang, L.; Liu, Z.; Chen, W., Pd x Cu 100- x networks: an active and durable electrocatalyst for ethanol oxidation in alkaline medium. Journal of Materials Chemistry A 2014, 2, 20933-20938.

(7) Lv, H.; Wang, Y.; Lopes, A.; Xu, D.; Liu, B., Ultrathin PdAg single-crystalline nanowires enhance ethanol oxidation electrocatalysis. Applied Catalysis B: Environmental 2019, 249, 116125.

(8) Zhong, J.; Bin, D.; Feng, Y.; Zhang, K.; Wang, J.; Wang, C.; Guo, J.; Yang, P.; Du, Y., Synthesis and high electrocatalytic activity of Au-decorated Pd heterogeneous nanocube catalysts for ethanol electro-oxidation in alkaline media. Catalysis Science \& Technology 2016, 6, 5397-5404. 